\title{
The RICH detector of the AMS-02 experiment: status and physics prospects
}

\author{
RUI PEREIRA, on behalf of the AMS RICH collaboration \\ $L I P / I S T$ \\ Av. Elias Garcia, 14, $1^{\circ}$ andar, 1000-149 Lisboa, Portugal \\ e-mail: pereira@lip.pt
}

\begin{abstract}
The Alpha Magnetic Spectrometer (AMS), whose final version AMS-02 is to be installed on the International Space Station (ISS) for at least 3 years, is a detector designed to measure charged cosmic ray spectra with energies up to the $\mathrm{TeV}$ region and with high energy photon detection capability up to a few hundred $\mathrm{GeV}$. It is equipped with several subsystems, one of which is a proximity focusing $\mathrm{RICH}$ detector with a dual radiator (aerogel $+\mathrm{NaF}$ ) that provides reliable measurements for particle velocity and charge. The assembly and testing of the AMS RICH is currently being finished and the full AMS detector is expected to be ready by the end of 2008. The RICH detector of AMS-02 is presented. Physics prospects are briefly discussed.
\end{abstract}

\section{The AMS-02 experiment}

The Alpha Magnetic Spectrometer (AMS), ${ }^{1}$ whose final version AMS-02 is to be installed on the International Space Station (ISS) for at least 3 years, is a detector designed to study the cosmic ray flux by direct detection of particles above the Earth's atmosphere, at an altitude of $\sim 400 \mathrm{~km}$, using state-of-the-art particle identification techniques. AMS-02 is equipped with a superconducting magnet cooled by superfluid helium. The spectrometer is composed of several subdetectors: a Transition Radiation Detector (TRD), a Time-of-Flight (ToF) detector, a Silicon Tracker, Anticoincidence Counters (ACC), a Ring Imaging Čerenkov (RICH) detector and an Electromagnetic Calorimeter (ECAL). A preliminary version of the detector, AMS-01, was successfully flown aboard the US space shuttle Discovery in June 1998.

The main goals of the AMS-02 experiment are: (i) a precise measurement of charged cosmic-ray spectra in the rigidity region between $\sim 0.5 \mathrm{GV}$ and $\sim 2 \mathrm{TV}$ and the detection of photons with energies up to a few hundred $\mathrm{GeV}$; (ii) a search for heavy antinuclei $(Z \geq 2)$, which if discovered would signal the existence of cosmological antimatter; (iii) a search for dark matter 
2
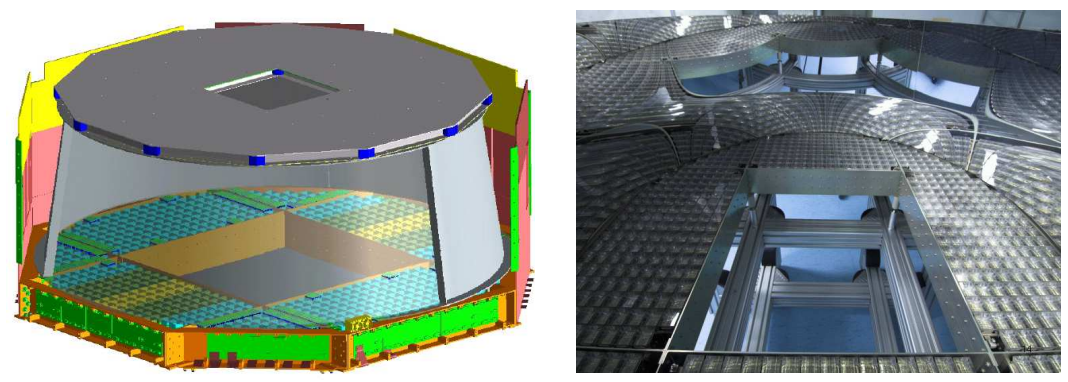

Fig. 1. The RICH detector of AMS-02 (left). View of the assembled RICH detector at CIEMAT (right).

constituents by examining possible signatures of their presence in cosmic ray spectra. The long exposure time and large acceptance $\left(0.5 \mathrm{~m}^{2} \cdot \mathrm{sr}\right)$ of AMS-02 will enable it to collect an unprecedented statistics of more than $10^{10}$ nuclei.

\section{The AMS RICH detector}

AMS-02 includes a proximity focusing Ring Imaging Čerenkov (RICH) detector, shown in Fig. 1, placed in the lower part of the spectrometer between the lower ToF counters and the ECAL. A dual radiator configuration with silica aerogel ( $n=1.050$ ) and sodium fluoride (NaF, $n=1.334$ ) has been chosen for the RICH. The geometrical acceptance of the central NaF square corresponds to $\sim 10 \%$ of the total RICH acceptance.

A high reflectivity $(>85 \%$ at $\lambda=420 \mathrm{~nm}$ ) lateral conical mirror made of aluminium-nickel-coated silica has been included to increase photon collection. The detection matrix at the bottom of the detector has 680 multianode photomultiplier tubes $(\mathrm{PMTs})(4 \times 4)$ coupled to light guides with a pixel size of $8.5 \mathrm{~mm}$.

Charged particles crossing a radiator of refractive index $n$ with a velocity $v>c / n$ emit Cerenkov radiation in a cone with an aperture $\theta_{c}=\arccos \left(\frac{1}{\beta n}\right)$. The intensity of radiation is proportional to the square of particle charge and also increases with velocity. The impact of photons in the PMT matrix produces a ring which, combined with data on particle tracks obtained from the Silicon Tracker, allows to determine the Čerenkov angle $\theta_{c}$ and therefore the particle's velocity. Particle charge may be determined from the total signal collected by the PMTs taking ring acceptance into account.

The analysis of RICH data involves the identification of the Čerenkov ring in a hit pattern which usually includes several scattered noise hits and an eventual strong spot in the region where the charged particle crosses the 
detection plane.

The RICH detector will provide a very accurate velocity measurement (in aerogel, $\Delta \beta / \beta \sim 10^{-3}$ and $10^{-4}$ for $Z=1$ and $Z=10-20$, respectively) and charge identification of nuclei up to iron $(Z=26)$. RICH data, combined with information on particle rigidity from the AMS Silicon Tracker, enable the reconstruction of particle mass. The accuracy of the RICH velocity measurement is essential for mass reconstruction due to the growth of relative errors when $\beta \sim 1\left(\frac{\Delta m}{m}=\frac{\Delta p}{p} \oplus \gamma^{2} \frac{\Delta \beta}{\beta}\right)$. The RICH detector also plays a major role in the exclusion of upward going particles (albedo). Additional information on the RICH detector may be found in Ref. 3. The assembly of the AMS RICH detector is coming to an end at CIEMAT in Madrid. The integration of the RICH and the other subdetectors of AMS-02 will take place at CERN and should be finished by the end of 2008 .
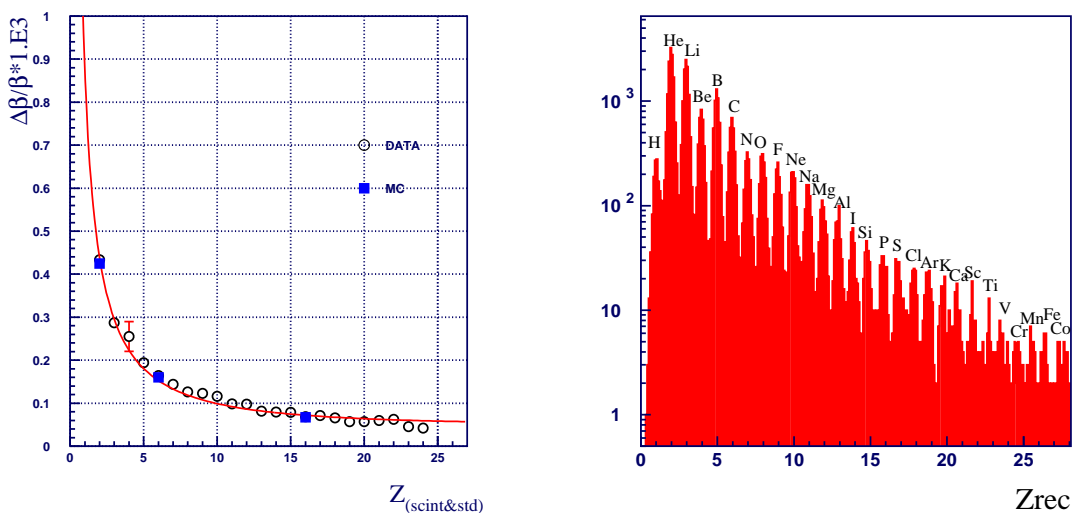

Fig. 2. Results from RICH prototype tests: velocity resolution (left) and charge distribution (right) for aerogel events.

\section{RICH prototype tests}

Tests on RICH particle detection were performed using a prototype corresponding to a fraction of the final detector (96 PMTs). The first prototype tests were performed in mid-2002 at LPSC in Grenoble by exposing the detector to the cosmic-ray flux at ground level. Later, in-beam testing took place at CERN SPS in October 2002 and October 2003 using secondary beams of nuclear fragments. ${ }^{2}$ The 2002 beam was produced by bombarding a Be target with $\mathrm{Pb}$ ions having a momentum of $20 \mathrm{GeV} / \mathrm{c} /$ nucleon, while the 2003 beam was obtained from the bombardment of a $\mathrm{Pb}$ target with In ions having a momentum of $158 \mathrm{GeV} / \mathrm{c} /$ nucleon. During the 2003 tests a 
mirror segment corresponding to a $30^{\circ}$ sector ( $1 / 12$ of total) was included in the experimental setup.

Fig. 2 shows prototype results for velocity and charge reconstruction with the aerogel radiator chosen for the final detector. A velocity resolution $\Delta \beta / \beta=8.7 \times 10^{-4}$ for $Z=1$ and $\Delta \beta / \beta \simeq 0.6 \times 10^{-4}$ for $Z>20$ was found. A good agreement between data and Monte Carlo results was observed. Charge separation was possible up to $Z \sim 26$, with a charge resolution $\Delta Z=0.16$ charge units for low $Z$. Further details on velocity and charge reconstruction can be found in Ref. 4 .

\section{System monitoring and pre-assembly testing}

A detailed monitoring of all components of the RICH detector is necessary to ensure that systematic errors are at the level required for good velocity and charge reconstruction. A good velocity measurement requires a knowledge of the aerogel's refractive index with a precision of $10^{-4}$. The quality of charge measurement constrains several variables: required knowledge of detector properties is at the level of $0.4 \mathrm{~mm}$ for aerogel thickness, $3 \%$ for aerogel clarity, $1 \%$ for mirror reflectivity and $5 \%$ for PMT gain and unit cell efficiency. Extensive testing has been performed on the RICH detector and its individual components to fulfill these requirements. A detailed mapping of each aerogel tile's thickness and refractive index was performed at LPSC in Grenoble with participation from LIP and UNAM. Mirror reflectivity was measured for several incidence angles. Studies for the characterization of PMTs and unit cells took place at CIEMAT.

Additional tests focused on the response of detector components to the conditions expected to occur during detector transportation into orbit and operation at the ISS. One rectangular grid, corresponding to approximately one-fifth of the detection matrix, underwent magnetic field testing at CERN and at LCMI in Grenoble to determine if PMT response would change significantly under a stray magnetic field of up to $300 \mathrm{G}$. Thermal and vacuum testing, which included individual PMTs being subject to temperatures between $-35^{\circ} \mathrm{C}$ and $55^{\circ} \mathrm{C}$ and a rectangular grid undergoing temperatures between $-20^{\circ} \mathrm{C}$ and $40^{\circ} \mathrm{C}$, took place at CIEMAT. Vibration tests of the radiator container (including all $\mathrm{NaF}$ tiles and $1 / 4$ of aerogel tiles) took place at SERMS in Terni, while one rectangular grid underwent similar testing at INTA in Madrid. Individual unit cells also underwent vibration tests. Monitoring and testing will continue during the detector assembly period. 

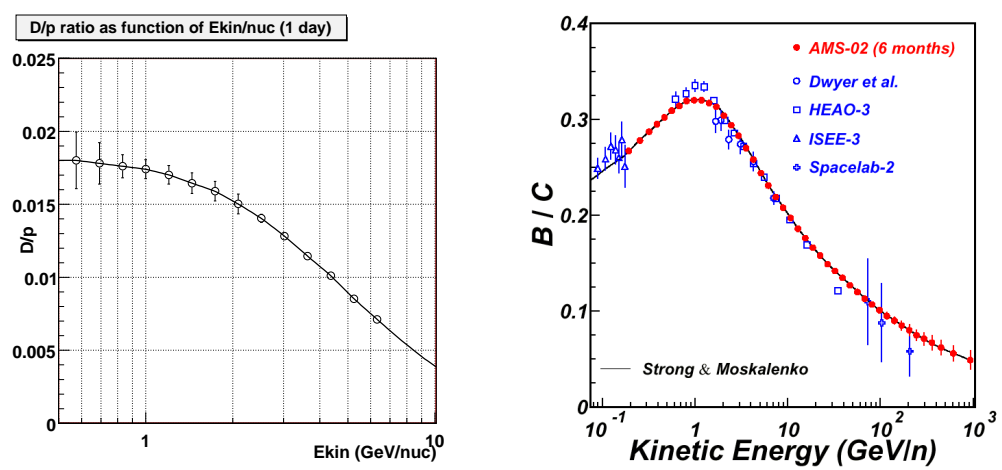

Fig. 3. Expected sensitivity of AMS: D/p ratio with one day of data (left) and B/C ratio with six months of data (right).

\section{Physics prospects}

The capacities of the RICH detector for velocity and charge reconstruction will play a major role in particle identification with AMS-02. RICH data will provide charge separation up to $Z \sim 26$. Mass measurements obtained from the combination of RICH velocity with Tracker rigidity data will make possible the isotopic separation of light elements such as $\mathrm{H}$, He and Be.

AMS data will provide new insights on cosmic ray physics. Secondary to primary ratios such as $\mathrm{D} / \mathrm{p},{ }^{3} \mathrm{He} /{ }^{4} \mathrm{He}$ and $\mathrm{B} / \mathrm{C}$ will provide information on cosmic ray propagation, while the ratio between the abundances of radioactive isotope ${ }^{10} \mathrm{Be}$ and stable ${ }^{9} \mathrm{Be}$ will provide data on galactic confinement times and help improve the accuracy of existing halo models.

\section{Conclusions}

AMS-02 will provide a new insight on the cosmic-ray spectrum by collecting precise data for an unprecedented number of particles above the Earth's atmosphere. The RICH detector will play a key role in the operation of AMS due to its capabilities for velocity reconstruction, charge determination and albedo rejection. Extensive testing has been performed on the RICH detector and its components. The assembly of the RICH detector is currently being finished and the full AMS detector is expected to be ready by the end of 2008 .

\section{References}

1. S. P. Ahlen et al., Nucl. Instrum. Methods A 350, 34 (1994).

V. M. Balebanov et al., AMS proposal to DOE, approved April 1995.

2. P. Aguayo et al., Nucl. Instrum. Methods A 560, 291 (2006).

3. F. Barao et al., Proceedings of 29th ICRC, Pune, India, 349-352 (2005), arXiv:astro-ph/0603852.

4. F. Barao et al., Proceedings of 30th ICRC, Merida, Mexico (2007), arXiv:0709.2154 [astro-ph]. 